\title{
Conclusions and Suggestions on Low-Dose and Low-Dose Rate Radiation Risk Estimation Methodology
}

\author{
Kazuo Sakai ${ }^{1}$, Yutaka Yamada ${ }^{2}$, Kazuo Yoshida ${ }^{3}$, Shinji Yoshinaga ${ }^{4}$, Kaoru Sato ${ }^{5}$, Hiromitsu Ogata ${ }^{6}$, Toshiyasu Iwasaki ${ }^{3}$, \\ Shin'ichi Kudo ${ }^{7}$, Yasuki Asada ${ }^{8}$, Isao Kawaguchi ${ }^{9}$, Hiroshi Haeno ${ }^{10}$, Michiya Sasaki ${ }^{3}$ \\ ${ }^{1}$ Higashigaoka Faculty of Nursing, Tokyo Healthcare University, Tokyo, Japan; ${ }^{2}$ Department of Radioecology and Fukushima Project, Center for Advanced \\ Radiation Emergency Medicine, Quantum Medical Science Directorate, National Institutes for Quantum and Radiological Science and Technology, Chiba, \\ Japan; ${ }^{3}$ Radiation Safety Research Center, Nuclear Technology Research Laboratory, Central Research Institute of Electric Power Industry, Komae, Japan; \\ ${ }^{4}$ Department of Environmetrics and Biometrics, Research Institute for Radiation Biology and Medicine, Hiroshima University, Hiroshima, Japan; \\ ${ }^{5}$ Environment and Radiation Sciences Division, Nuclear Science and Engineering Center, Japan Atomic Energy Agency, Tokai-mura, Japan; ${ }^{6}$ Graduate School \\ of Nutrition Sciences, Kagawa Nutrition University, Sakado, Japan; ${ }^{7}$ Institute of Radiation Epidemiology, Radiation Effects Association, Tokyo, Japan; \\ ${ }^{8}$ Deviation of Medical Physics, Faculty of Radiological Technology, School of Medical Sciences, Fujita Health University, Toyoake, Japan; ${ }^{9}$ Center for Radiation \\ Protection Knowledge, National Institute of Radiological Sciences, National Institutes for Quantum and Radiological Science and Technology, Chiba, Japan; \\ ${ }^{10}$ Department of Computational Biology and Medical Sciences, Graduate School of Frontier Sciences, The University of Tokyo, Kashiwa, Japan
}

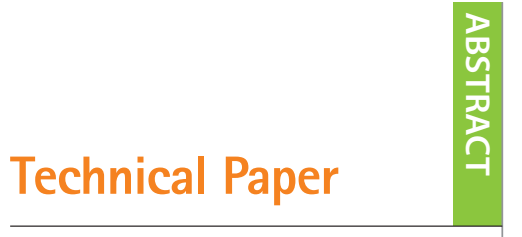

Received November 27, 2020

Revision January 25, 2021

Accepted February 1, 2021

Corresponding author: Michiya Sasaki

Central Research Institute of Electric Power Industry, 2-11-1 Iwado kita, Komae, Tokyo 201-8511, Japan

E-mail:michiya@criepi.denken.or.jp

(DiD https://orcid.org/0000-0002-0444-5459

This paper is an invited paper recommended by the Japan Health Physics Society (JHPS).

This is an open-access article distributed under the terms of the Creative Commons Attribution License (http://creativecommons.org/licenses/by-nc/4.0), which permits unrestricted use, distribution, and reproduction in any medium, provided the origina work is properly cited.

Copyright $\odot 2021$ The Korean Association for Radiation Protection

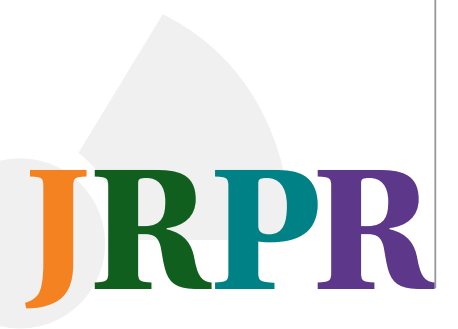

www.jrpr.org
Background: For radiological protection and control, the International Commission on Radiological Protection (ICRP) provides the nominal risk coefficients related to radiation exposure, which can be extrapolated using the excess relative risk and excess absolute risk obtained from the Life Span Study of atomic bomb survivors in Hiroshima and Nagasaki with the dose and dose-rate effectiveness factor (DDREF).

Materials and Methods: Since it is impossible to directly estimate the radiation risk at doses less than approximately $100 \mathrm{mSv}$ only from epidemiological knowledge and data, support from radiation biology is absolutely imperative, and thus, several national and international bodies have advocated the importance of bridging knowledge between biology and epidemiology. Because of the accident at the Tokyo Electric Power Company (TEPCO)'s Fukushima Daiichi Nuclear Power Station in 2011, the exposure of the public to radiation has become a major concern and it was considered that the estimation of radiation risk should be more realistic to cope with the prevailing radiation exposure situation.

Results and Discussion: To discuss the issues from wide aspects related to radiological protection, and to realize bridging knowledge between biology and epidemiology, we have established a research group to develop low-dose and low-dose-rate radiation risk estimation methodology, with the permission of the Japan Health Physics Society.

Conclusion: The aim of the research group was to clarify the current situation and issues related to the risk estimation of low-dose and low-dose-rate radiation exposure from the viewpoints of different research fields, such as epidemiology, biology, modeling, and dosimetry, to identify a future strategy and roadmap to elucidate a more realistic estimation of risk against low-dose and low-dose-rate radiation exposure.

Keywords: Radiation Risk Estimation, Low Dose and Low Dose Rate, Epidemiology, Biology, Modeling, Dosimetry 


\section{Introduction}

Radiation risks have been estimated mainly on the basis of epidemiological data $[1,2]$. However, for doses lower than $100 \mathrm{mSv}$, it is difficult to evaluate the risks quantitatively because of the statistical limitation of epidemiological results. Larger cohort and longer follow-up period are needed to detect health effects by low-dose/low-dose-rate radiation compared with high-dose/high-dose-rate radiation. In addition to this, obtaining the information other than radiation such as smoking habit are difficult, in case the study was retrospective cohort study. Due to this lack of information, there is a possibility that the adjustment for confounding factors is insufficient. Radiation biology is expected to help overcome this limitation; it has provided much knowledge of radiation effects associated with radiation carcinogenesis but not much on matters that can directly support the improvement of quantitative risk inference in humans.

After the accident at the Tokyo Electric Power Company (TEPCO)'s Fukushima Daiichi Nuclear Power Station in 2011, the situation of low-dose-rate radiation exposure suddenly appeared in Japanese communities, which attracted the interest in radiation risk from the public. The exposure level after the accident was several tens of times higher than the natural background radiation level under ordinary situations. However, on the basis of the reports of the United Nations Scientific Committee on the Effects of Atomic Radiation (UNSCEAR) and the World Health Organization [3, 4], the dose and dose-rate are still classified as low, and the effects and inferred excess risk are assessed to be very small even then radiation can be detected.

The risk inference at low-dose and/or low-dose-rate contains uncertainties, because it is extrapolated from the results at higher dose/dose-rate, such as the results of the epidemiological study of atomic bomb survivors of Hiroshima and Nagasaki. Sources of uncertainty in risk estimate and risk assessment are described and analyzed by the National Council on Radiation Protection and Measurements (NCRP) in detail, including dosimetric uncertainties, epidemiological and methodological uncertainties, uncertainties from low statistical power and precision, uncertainties from inadequate modeling of radiation risk data, and transport of (or generalizing) risk estimates to different populations [5].

On the other hand, risk inference in the case of an existing exposure situation (e.g., after a nuclear hazard) should be realistic, whereby the methodology of risk estimation needs to consider many factors related to health risks other than radiation and not the conventional conservative ones that only focus on radiological protection. For this purpose, it is necessary to solve many multidisciplinary issues.

Recently, several domestic or international activities have been initiated to improve risk estimation at low-dose and/or low-dose-rate. In Japan, NIRS-QST (National Institute of Radiological Sciences, a part of the National Institutes for Quantum and Radiological Sciences and Technology) organized the Radiation Risk and Protection Research Platform Preparatory Committee in 2016, and this preparatory committee summarized the functional requirements for the research platform for low dose and/or low dose rate radiation risk estimation. Then the Planning and Acting Network for Low Dose Radiation Research (PLANET) started actions in 2017. In Europe, the Multidisciplinary European Low Dose Initiative (MELODI) was founded in 2010. Recently, radiation-related platforms in Europe, such as the European Radiation Dosimetry Group (EURADOS), the European Platform on Preparedness for Nuclear and Radiological Emergency Response and Recovery (NERIS), the European Alliance for Medical Radiation Protection Research (EURAMED), have held joint workshops named the European Radiation Protection Week since 2016 and tried to integrate their activities. In the United States, the Electric Power Research Institute (EPRI) launched the International Dose Effect Alliance (IDEA), and NCRP summarized the approaches for integrating information from radiation biology and epidemiology by reviewing the available biologically based dose-response (BBDR) model and research needs for defining adverse outcome pathways (AOPs) [6]. All these activities aim to improve risk estimation at low doses and/or low-dose rates by a multidisciplinary approach.

We established the research group on low-dose and lowdose-rate radiation risk estimation methodology, with the permission of the Japan Health Physics Society (JHPS). The aim of this research group was to clarify the current situation and issues related to the risk estimation of low-dose and low-dose-rate radiation exposure, while reviewing important studies by experts from four research fields, namely, epidemiology, biology, modeling, and dosimetry. We also compared the current situation in the field of risk evaluation of chemicals, including AOPs. The research group worked from April 2016 to March 2018, holding eight expert meetings and sessions at the annual meeting of the JHPS during that period, and finally summarized the report. In this paper, we 
briefly describe the conclusions and suggestions from its final summary report published in September 2019 on the JHPS website.

\section{Materials and Methods}

\section{Epidemiological Research}

In the field of radiological protection, the most notable epidemiological study is the follow-up study of atomic bomb survivors in Hiroshima and Nagasaki (Life Span Study) conducted by the Radiation Effects Research Foundation. The main results were applied for the assessment of the lifetime risk of cancer mortality, cancer incidence (nominal population risk), or detriment calculations by UNSCEAR and the International Commission on Radiological Protection (ICRP). This lifetime risk has been adopted as the main basis for the weighting factor and is used for expert judgment of the scale indicating the effects of radiation exposure. Dose response curves and parameters derived from statistical analysis are also used for the quantitative evaluation of low dose or dose rate effects [7].

Although, epidemiological studies on atomic bomb survivors basically observe the health effects of a single (shorttime) exposure at a high-dose rate, the health risk of low-dose rates and long-term radiation exposure is not clearly observed. Therefore, it is not appropriate to use those results directly. On the other hand, many epidemiological studies on the health effects of low-dose and low-dose-rate radiation have been conducted, where people are likely to be exposed in various situations, such as the environment, occupation, and medical care. As for the risk assessment of low-dose and lowdose-rate radiation, the uncertainty is large and does not provide a direct basis. Therefore, the risk assessment of lowdose and low-dose-rate radiation for radiological protection relies on extrapolation from high-dose and high-dose-rate exposure data using a linear no-threshold (LNT) model.

The reasons for the large uncertainties in the risks of lowdose and low-dose-rate radiation include insufficient statistical power, uncertainty in dosimetry, and insufficient adjustment of confounding factors. Regarding the health effects of low-dose and low-dose-rate radiation, social concerns have been particularly growing since the Fukushima Daiichi Nuclear Power Plant accident in 2011, but as described above, results from the epidemiological studies cannot be adopted directly. Uncertainties in the findings are large and have not yet reached social consensus.
In light of the above, we will first present a representative epidemiological report that has recently received attention. Selected from the last 5 years or so, overview, criticism, and the issues in future epidemiological research are summarized as follows (See [8] for details).

The sources of uncertainty in low-dose radiation risk estimation from an epidemiological point of view include poor statistical power and insufficient adjustment of confounders. In order to compensate for the lack of power, 15 countries analysis and joint analysis such as INWORKS have been performed $[9,10]$. However, the issue of confounding factor adjustment remains, and few studies have adjusted for smoking, which has a particularly large effect on mortality [11-13].

In order to solve these uncertainties, it is desired to perform a pool analysis that excludes confounding factors by adjusting for smoking and similar factors to form a larger population. However, there is a limit to estimating the risk of low-dose radiation based solely on the results of conventional epidemiological studies, even if the effects of confounding factors are reduced and the power is significantly improved. Therefore, closer collaboration between epidemiological and biological research is desired. Specifically, the following approaches are necessary to incorporate biological techniques and knowledge into epidemiological research.

(1) Collect temporal information and biological samples related to exposure, health effects, and confounding factors among others during the course of disease development from exposure.

(2) Apply knowledge/techniques such as molecular biology to assess exposure-related chromosomal abnormalities and DNA double-strand break markers such as $\gamma \mathrm{H} 2 \mathrm{AX}$ using collected biological samples as well as genetic factors such as genetic polymorphisms to evaluate the effect of the genetic modification.

(3) Analyze dose-response relations such as linear and quadratic, the effects of age modification on dose-response relations, and the role of confounding factors based on biological mechanisms.

(4) Utilize information on exposure factors other than radiation and biological samples among others in cooperation with other large-scale research studies such as genome cohort research and eco-chill research.

(5) Analyze the relationship between exposure and health effects on the basis of state-of-the-art information science and statistics such as Bayesian techniques.

Considering the above, it is expected that research will fur- 
ther integrate epidemiological and biological knowledge, and will enable a more accurate estimation of radiation risk at low doses and low dose rates.

\section{Biological Research}

ICRP recommends that the dose and dose-rate effectiveness factor (DDREF) value is 2 from the analysis of atomic bomb survivor data, other epidemiology data, and specific animal experiment data [1], while the National Academy of Sciences Biologic Effects of Ionizing Radiation (BEIR) VII report indicates the value of 1.5 [7]. In a recent study, the estimation of DDREF is attempted using risks presumed on account of a study (meta-analysis of the nuclear worker) of protracted radiation exposure being compared with the study (atom bomb survivor) of high-dose-rate radiation exposure. As a result, the ratio is shown to become less than $1[14,15]$.

As exposure dose and dose rate can be controlled in animal experiments unlike in epidemiological studies, the use of animal data is useful for the estimation of DDREF, although species difference should be considered. The animal experiment data are maintained as databases (Northwestern University Radiation Tissue Archives [NURA], and European Radiobiological Archive [ERA]), and studies to evaluate DDREF have been carried out by the reanalysis of the data [16, 17]. In the reanalysis, endpoints are divided into cancer and noncancer diseases, and the effects at the organ level are evaluated. It is reported that the dose-rate effectiveness factor (DREF) of many endpoints at the low-dose-rate radiation (less than $5 \mathrm{mGy} / \mathrm{h}, 120 \mathrm{mGy} /$ day) is in the range of 1.2-2.3, and it is not markedly different from the result of the epidemiological study.

There is an advantage of analyzing human data directly in the epidemiological study, but there is difficulty in evaluating the confounding factor. On the other hand, in biological research by molecular, cellular, and animal experiments, one can investigate radiation effects and their mechanisms. It is, however, usually difficult to apply the results from animal experiments to humans. Thus, these two approaches, epidemiological study and biological research, are complementary, and it is considered that a general understanding of the risk of low-dose and low-dose-rate radiation can be achieved.

The three following elements are required to estimate the risk of low-dose and low-dose-rate radiation.

(1) Epidemiological study of low-dose and low-dose-rate radiation designed for an appropriate risk evaluation: It is questionable whether a LNT model is applicable in a region of low-dose exposure, and whether the value of DDREF is appropriate as expected. As a result, the risk estimate obtained from the epidemiological study includes an uncertainty $[14,15]$. An epidemiological study that is designed to lower the uncertainty as much as possible is necessary.

(2) Mechanism elucidation study for risk estimation of low-dose and low-dose-rate radiation: it is necessary to elucidate mechanisms underlying the low-dose and low-dose-rate radiation-induced biological effects. For example, it is thought that accumulation of mutations or chromosome aberrations due to the early DNA damage in tissue stem cells causes carcinogenesis [18]. The themes of biological research that should be carried out are as follows:

a) At the individual level of animals, the risk estimation of carcinogenesis and the study of its modifying factors, such as sex, age, genetic background, epigenetic factor, lifestyle, and combined effects of radiation and chemicals.

b) At the cellular level, the study of carcinogenesis in target stem cells, such as the dynamics of stem cells and the interaction between stem cells and the surrounding micro-environments.

c) At the molecular level, the study of bioindicators concerned with carcinogenesis, such as mutation, epigenetic change, redox state, genetic polymorphism, DNA repair, apoptosis, immune status, endocrine function, and inflammatory condition.

(3) Interpretation research using animal experiment data to interpret the results of epidemiological study: On the basis of the knowledge provided in a mechanism study of the expression of radiation effects, proof data accumulated by a biological approach mainly on animal experiments are used for the data interpretation in epidemiologic studies utilizing mathematical models.

It is necessary that the pathway by which the radiation exposure leads to biologically meaningful effects is clarified by the knowledge that these studies provide. In the ecotoxicology research field, the toxic effects of chemical substances are summarized, and AOPs are devised as a conceptual framework to support risk assessment. It is thought that this AOP is being applied to the evaluation of the radiation effects. In that case, molecular initiating events (MIEs) include DNA strand breaks, DNA base damage, protein modification, free radical generation, chromosome aberration, and so forth. 
Key events (KEs) include activation of gene expression, protein production, alteration of intracellular signaling, and so forth. These events become the index that can be measured quantitatively as a biomarker or a bioindicator. Cancer and a non-cancer diseases are added to the adverse outcome (AO) of radiation exposure as the individual response, such as death, oncogenic abnormality, and deterioration of reproduction.

However, AOPs are limited to those where AO always occurs KEs and their relationship with the low-dose and lowdose-rate radiation may be different from those with the high-dose radiation For example, DNA repair may not be caused by the low-dose and low-dose-rate radiation. As a result, it may vary in those AOPs. Therefore, it is necessary to clarify the specificity of KEs and AOPs in the low-dose and low-dose-rate radiation.

\section{Current Situation and Issues of Modeling Research}

Risk assessment is a scientific approach, and the results can provide scientific evidence in risk management. However, in order to connect the results of these risk assessments to actual risk management, it is necessary to discuss its appropriateness not only from a scientific perspective, but also from various aspects, such as social or policy perspectives. In such discussions, the scientific evidence provided by risk assessment has two main directions. One is to provide multiple scientific options with different viewpoints, and the other is to provide integrated lines of evidence by some scientific method. The former focuses on investigating many factors related to risk and quantitatively predicting the results of each factor, such as effects and interactions. These results provide different options because the viewpoints differ depending on the combination of factors and the method of data collection (e.g., experimental or epidemiological methods). On the other hand, the latter aims to provide a common framework for rational discussion after clarifying differences in perspectives and methodologies regarding risk esti- mation. In any of these directions, "models" play an important role.

One of the roles of models is to represent a quantitative relationship between dose and risk on the basis of epidemiological or experimental data. Furthermore, incorporating other elements into the model will lead to the refinement of risk estimation and mechanism elucidation. When building a model, it is necessary to make various types of assumptions, and in order to evaluate the validity of this model, it is necessary to discuss several aspects depending on the assumptions.

In general, there are models based on statistical data (hereinafter referred to as epidemiological models) and models that consider biological mechanisms (hereinafter referred to as biological models). Epidemiological models are mainly used for epidemiological data, and biological models are mainly used for experimental data. The former can deal directly with data on human populations, but it is difficult to elucidate biological mechanisms owing to phenomenological interpretation or probabilistic inference. On the other hand, since the latter is constructed on the basis of biological knowledge, its great advantage is that the biological interpretation can be directly performed on the estimated parameters. In any model, the mechanism and phenomenon are approximated, because there are still many points that cannot be fully explained concerning the mechanisms underlying the complex biological effects of radiation. However, the both roles of models are common since that they are developed quantitatively on the basis of data, and there are some overlaps in the analysis method (Table 1, Fig. 1).

The main role of epidemiological models is to quantitatively express the relationship between radiation exposure and risk from a phenomenological viewpoint. That is, many epidemiological studies use models to estimate dose-response relationships. The advantage of epidemiological models is that they take into account statistical uncertainties, such as data variability and variance of estimates. There are several

Table 1. Main Features of Statistical and Biological Models

\begin{tabular}{lcl}
\hline Model & \multicolumn{1}{c}{ Main data } & Use or build a model \\
\hline Epidemiological models & $\begin{array}{c}\text { Epidemiological data (observational study } \\
\text { on human population) }\end{array}$ & $\begin{array}{l}\text { Parameter estimation for existing models } \\
\text { Application of new methods in statistics } \\
\text { Biological models }\end{array}$ \\
& $\begin{array}{l}\text { Experimental data (animals, tissues, cells, } \\
\text { molecules, genes, etc.) }\end{array}$ & $\begin{array}{l}\text { Presentation of new indicators or new models } \\
\text { Model combination }\end{array}$ \\
& & $\begin{array}{l}\text { Parameter estimation } \\
\text { Adding parameters to an existing model }\end{array}$ \\
\end{tabular}




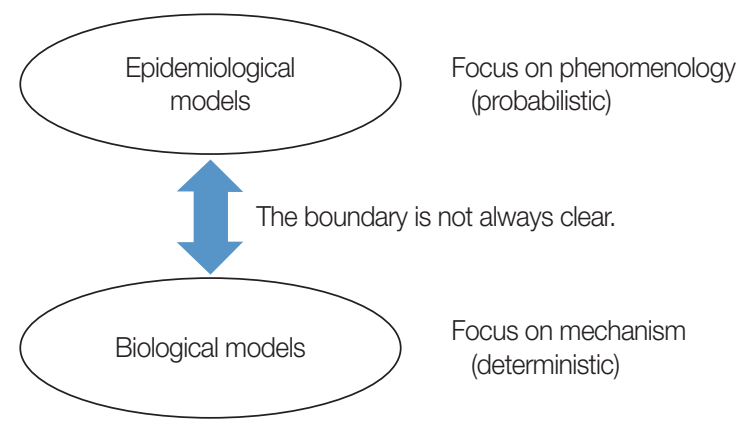

Fig. 1. Concept of differences between the two models.

epidemiological models for estimating dose-response relationships, but the main ones are Poisson regression, logistic regression, proportional hazard model, and so on. The risk indicators used in these models include relative risk (RR), excess relative risk (ERR), hazard ratio, and odds ratio.

Since biology is the subject of research from the micro- to the macro-levels, modeling is performed for each level. Intracellular levels are often deterministic in describing reactions in a relatively short period of time and are not suitable for modeling where irradiation and reaction take more than a year, such as radiation-induced carcinogenesis. These results are used as part of model parameters that are closer to the individual level. In addition, biological models are used to model changes over time; therefore, dose rates can be explicitly incorporated into the model. This is a bottom-up approach because the mechanism is explicitly modeled as a causal structure. In the future, in order to quantitatively evaluate the risks of low-dose and low-dose-rate radiation, which are difficult to verify in epidemiology, we will introduce experimentally verified models considering biological mechanisms, and it is expected that these models will be utilized even if there are differences in parameters (e.g., dose, dose rate, line type, biological background, and exposure situation).

\section{Current Situation and Issues of Dosimetric Research}

ICRP defined protection quantities such as equivalent dose and effective dose, which are closely related to risks (e.g., cancer and heritable effects) from a radiation exposure [1]. The protection quantities are based on the absorbed dose in an organ (organ dose) and are used for the reduction and management of exposure doses to ensure that the radiation risks are kept below unacceptable levels. Since organ doses cannot be directly measured in an actual living person, organ doses have been evaluated by using human models. Instead of previous human models [19], which are mathematically described by simple equations, ICRP used reference human models [20] consisting of rectangular blocks called voxels (volume pixels) for calculating the organ doses. The organs and tissues in reference human models are accurately defined by using voxels. The reference human models have organ masses, heights, and weights taken from the averages of Caucasian as the reference values reported by ICRP, and were used to calculate the protection quantities for external radiation fields [21] and for the intake of radionuclides [21-25].

Conversion coefficient datasets $[17,19]$ are given for the idealized irradiation geometries and radiation fields and for the reference human models by ICRP. The idealized irradiation geometries may be taken as approximations to actual conditions of exposure. In epidemiological studies, the uncertainties in radiation detectors are evaluated. In addition, the organ doses may be evaluated using dose conversion coefficients based on radiation fields by an expert's decision $[26,27]$. Radiation weighting factors defined by ICRP are not assigned to every organ and are different from the relative biological effectiveness (RBE), which is an index representing the effect on biological endpoints [28]. In dose assessments for atomic bomb survivors in Hiroshima and Nagasaki, a value of 10 was adopted for a radiation weighting factor for neutrons given as a continuous function of neutron energy. The neutron doses were added to exposure doses derived from gamma rays [29].

In internal exposures, the organs are irradiated with the radiation from radionuclides incorporated into the human body. ICRP provided the dose coefficients [22-24] and specific absorbed fractions [25] using biokinetic models [22-24, 30,31 and nuclear decay data [32] of radionuclides and reference human models [20]. These data and models are prepared using available scientific information. However, all the data needed for dose assessment against human and radionuclides are not available. Thus, scientific information obtained from animal experiments and other radionuclides is also used in the construction of the above data and models [22]. In addition, the physical and chemical characteristics, metabolisms (rates of absorption or excretion, and retention time) and intake scenario (the availability of protection equipment, the pattern and mode of intake, and the elapsed time between intake and measurement) of radionuclides, and anatomy (shape, location and mass of organ) are the factors of doses resulting from internal exposure. By taking the individual differences into consideration, one may find that the uncertainty in internal dose assessment (assessment of in- 
take of radionuclides from monitoring data and calculation of committed equivalent dose and committed effective dose) is very large and is a factor of at least $3[33,34]$.

The organ dose, which is closely related to radiation risks, is calculated by averaging the deposited energy over an organ or a tissue volume [1]. In exposure resulting from radiation accident and radiation therapy, the distributions of dose may be heterogeneous. The heterogeneous distributions of doses can also be caused by anatomical characteristics, such as the distributions (or locations) of organs in the body (e.g., whole or partial body), the boundaries between organs with different densities (e.g., red bone marrow and cortical bone), and membranous structures (e.g., urinary bladder and gastrointestinal tract). The distributions of doses in microscopic target regions (e.g., cell, nucleus, and DNA) become heterogeneous with the decrease in dose or dose rate. In particular, target size and radiation type affect dose distribution.

In the future, the following research issues will probably be important for the improvement of low-dose and low-doserate radiation risk estimation: (1) RBE is obtained from experimental animal radiation studies and is dependent on the types of biological effect and radiation. When the organ doses due to neutron exposures in small animals (e.g., mouse) are extrapolated to those in humans, it is necessary to consider the differences in behavior of secondary radiation resulting from the differences in body sizes [35] and from species differences in radiosensitivity. (2) In internal exposures, the organs and tissues are irradiated with radiation from the radionuclides incorporated into body over time periods depending on their physical and biological half-lives. Thus, in internal dose assessments, the organ dose and effective dose are derived as the total dose delivered within a specified commitment period considering the behavior of radionuclides and their retention in the body [1]. The committed effective dose from internal exposure is regarded as the personal dose equivalent, while the dose rates could be different between the internal and external exposure. Therefore, the differences in the types of exposure may impact on the estimation of exposure doses using the biological samples or biomarkers as an index.

\section{Results and Discussion}

\section{Discussion of Possible Roadmap}

Issues and proposals for the construction of a low-dose and low-dose-rate radiation risk assessment method are dis- cussed in this section.

Models based on epidemiological data have long been used for the quantitative assessment of radiation risk. The LNT model is a typical example. However, most models based on epidemiological data are epidemiological models, and the parameters used therein are not associated with biological parameters. On the other hand, approaches to developing models based on biological mechanisms are still limited and have not reached the level at which they can be used for assessing human risk. In the future, the following two approaches can be considered to develop a model that can estimate low-dose and low-dose-rate radiation risks: (1) fusion of statistical and biological models, and (2) biological construction and verification of new models based on mechanisms.

\section{Fusion of Different Models}

"Fitness" to actual data is one of the main indicators for validity assessment in any model. Therefore, it is necessary to build a model with as few parameters as possible while considering both consistency with data and mechanisms. As for model compatibility, a statistical model should take into account biological mechanisms and a biological model should consider the phenomena of a human population. That is, in order to fuse models, it would be effective to quantitatively incorporate individual differences among human populations and differences between species into models that express biological mechanisms. On the other hand, it will be a challenge to remove as much as possible the effects of confounding factors and bias in epidemiological data by rational methods. In any case, model fusion may partially be possible by comparing the common elements of different models.

\section{Recommendations Regarding Activity Goals in Each Field}

In order to develop a low-dose and low-dose-rate radiation risk assessment method, studies and discussions involving various related research fields aiming at the following are considered effective.

(1) Epidemiology

- To eliminate noise thoroughly

- To not end up with both good and bad discussions on epidemiological models

(2) Biology

- To be quantitative

- To be parametric 


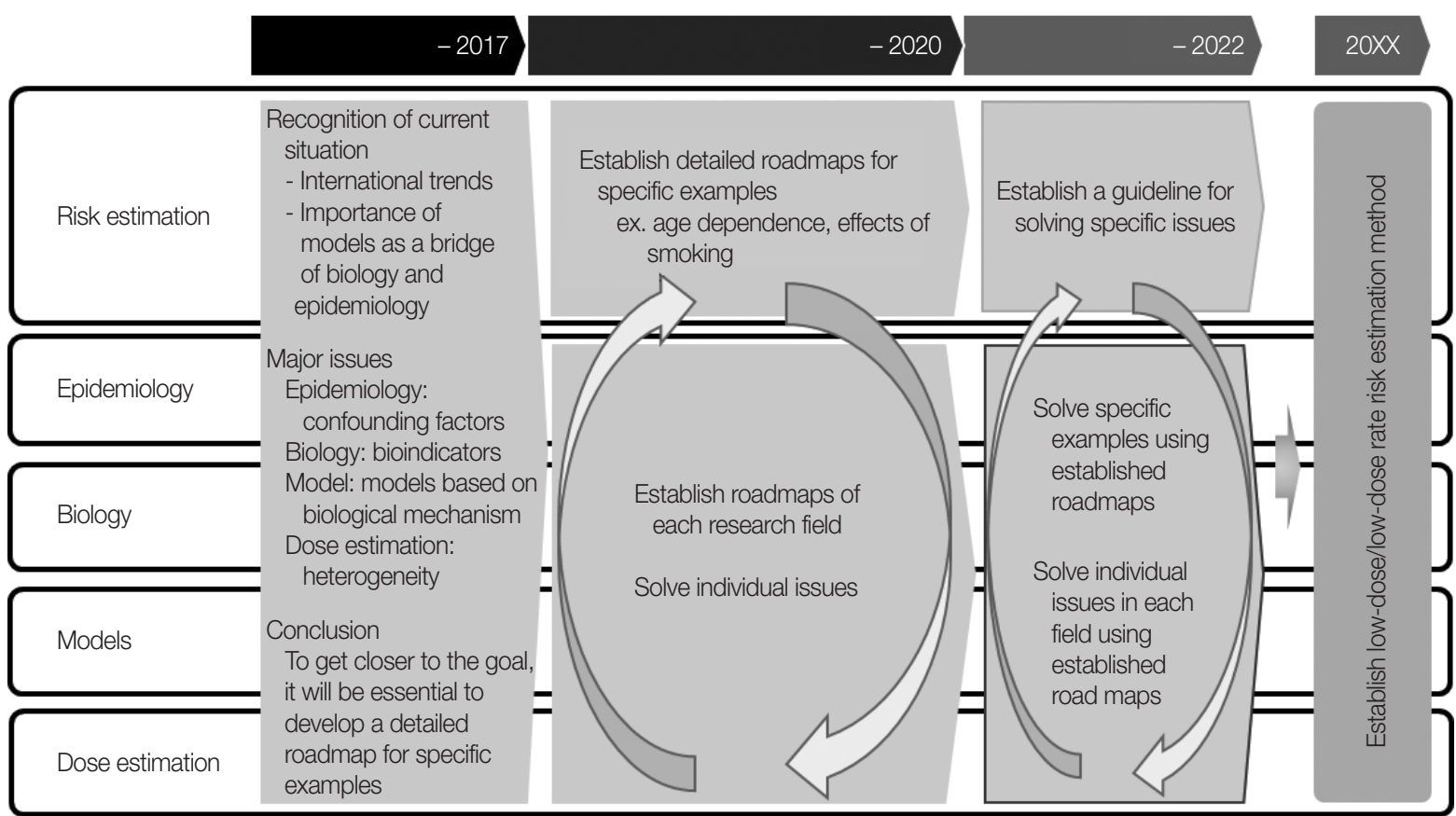

Fig. 2. Research roadmap for establishing low-dose and low-dose rate risk estimation methods developed by the research group.

(3) Model research

- To be applicable to verifying a model that connects the phenomena of human populations and biological mechanisms.

- To be applicable to estimating the low-dose and lowdose-rate risk of humans.

(4) Dose evaluation

- To be applicable to comparing doses for humans and animals on the same scale.

\section{Summary and Research Roadmap}

Fig. 2 shows the roadmap of research obtained through the activities of this research group. This research group clarified that there are a wide variety of issues in the fields of epidemiology, biology, models, and dose assessment. To get closer to the goal, it will be essential to develop a detailed roadmap for specific examples, to feed it back to the overall roadmap, and to discuss it with other academic societies, related organizations, and overseas experts.

\section{Conclusion}

The cliché that "the fusion of biology and epidemiology is important" for the method of estimating low-dose and lowdose-rate radiation risks has been proclaimed for nearly half a century. In the meantime, there has been an increase in the use of radiation, especially in the medical field, and unfortunately, Chernobyl and Fukushima Daiichi Nuclear Power Plant accidents also occurred, which has heightened public interest in radiation. Nevertheless, progress in the risk assessment has not been made because the mechanism of carcinogenesis, which are estimated as main risk for low-dose and low-dose-rate radiation exposure, has not been elucidated, and the contribution of radiation has not been discussed in detail. It is considered that various factors such as the difference in biological response to low-dose radiation and the weak biological response at low-doses make quantitative measurement difficult.

On the other hand, in recent years, it has become possible to observe the effects at the gene level owing to the development of DNA analysis technology and rapid test, and life science has undergone a transformation incomparable to that a decade ago. Regarding tissue stem cells, which are considered targets for radiation carcinogenesis, markers have been identified in multiple tissues, and it has become possible to observe various dynamics in vivo. In addition, efforts to quantitatively understand biological phenomena using mathematical models and the latest analytical instruments, such as those in mathematical biology and quantitative biology, have been greatly advanced.

The introduction of these latest technologies will be a powerful tool for elucidating the mechanism of action of ra- 
diation and quantifying it, especially in the field of radiation biology. The research group noted the importance of "model research" as a bridge between epidemiology and biology. There are various issues to overcome the limitation of the conventional radiation risk estimation, and the path to their solutions are not straightforward. At the very least, it will be necessary for experts in each field to maintain their interests in other research fields that are presently "incomprehensible" and to continue to search for collaborations without being confined to each field. The study group was able to obtain cross-disciplinary cooperation from experts in epidemiology, biology, model research, and dose assessment. The fact that such a cooperative system could be built could itself be a major achievement for the future. The next step, as indicated in this report, is to proceed the individual research.

\section{Conflict of Interest}

No potential conflict of interest relevant to this article was reported.

\section{Acknowledgements}

We thank members from various fields in addition to those from the conventional radiological protection field for their cooperation. In addition, useful discussions were made with collaborators and observers from other academic societies. We received much valuable information from radiological and chemical risk assessment experts. We would like to express our sincere appreciation to Dr. Nori Nakamura for his lecture on the bridge between biological research and epidemiology, Dr. Hajime Kojima for his lecture on the basics of the adverse outcome pathway with its current status and trends, and the JHPS Planning Committee for carefully reviewing the final report.

\section{Author Contribution}

Project administration: Sakai K, Yamada Y, Yoshida K, Sasaki M. Writing - original draft: Yamada Y, Yoshida K, Yoshinaga S, Sato K, Ogata H, Iwasaki T, Kudo S, Asada Y, Kawaguchi I, Haeno H, Sasaki M. Writing - review \& editing: Sakai K, Yamada Y, Yoshida K, Yoshinaga S, Sato K, Ogata H, Iwasaki T, Kudo S, Asada Y, Kawaguchi I, Haeno H, Sasaki M. Investigation: Yoshinaga S, Sato K, Ogata H, Iwasaki T, Kudo S, Asada Y, Kawaguchi I, Haeno H. Supervision: Sakai K.

\section{References}

1. The 2007 Recommendations of the International Commission on Radiological Protection: ICRP publication 103. Ann ICRP. 2007;37:1-332.

2. United Nations Scientific Committee on the Effects of Atomic Radiation. Effects of ionizing radiation. Volume I: Report to the general assembly, scientific annexes A and B. Vienna, Austria: United Nations Scientific Committee on the Effects of Atomic Radiation; 2006.

3. United Nations Scientific Committee on the Effects of Atomic Radiation. Sources, effects and risks of ionizing radiation. Volume I: Report to the general assembly, scientific annex A. Vienna, Austria: United Nations Scientific Committee on the Effects of Atomic Radiation; 2013.

4. World Health Organization. Health risk assessment from the nuclear accident after the 2011 Great East Japan earthquake and tsunami, based on a preliminary dose estimation. Geneva, Switzerland: World Health Organization; 2013.

5. National Council on Radiation Protection and Measurements. Uncertainties in the estimation of radiation risks and probability of disease causation (NCRP Report No. 171). Bethesda, MD: National Council on Radiation Protection and Measurements; 2012.

6. National Council on Radiation Protection and Measurements. Approaches for integrating information from radiation biology and epidemiology to enhance low-dose health risk assessment (NCRP Report No. 186). Bethesda, MD: National Council on Radiation Protection and Measurements; 2020.

7. National Research Council. Health risks from exposure to low levels of ionizing radiation: BEIR VII, phase 2. Washington, DC: The National Academies Press; 2006.

8. Japan Health Physics Society. Reports of JHPS Research Group on low-dose and low-dose-rate radiation risk estimation methodology (vol. 11, no. 1) [Internet]. Tokyo, Japan: Japan Health Physics Society; 2019 [cited 2021 Feb 11]. Available from: http:// www.jhps.or.jp/upimg/files/report11.1.pdf.

9. Richardson DB, Cardis E, Daniels RD, Gillies M, O’Hagan JA, Hamra GB, et al. Risk of cancer from occupational exposure to ionising radiation: retrospective cohort study of workers in France, the United Kingdom, and the United States (INWORKS). BMJ. 2015;351:h5359.

10. Cardis E, Vrijheid M, Blettner M, Gilbert E, Hakama M, Hill C, et al. The 15-country collaborative study of cancer risk among radiation workers in the nuclear industry: estimates of radiationrelated cancer risks. Radiat Res. 2007;167:396-416.

11. Kudo S, Ishida J, Yoshimoto K, Ohshima S, Furuta H, Kasagi F. The adjustment effects of confounding factors on radiation risk estimates: findings from a Japanese epidemiological study on low-dose radiation effects (J-EPISODE). J Mol Genet Med. 2017; 


\section{1:1000275.}

12. Gilbert ES, Sokolnikov ME, Preston DL, Schonfeld SJ, Schadilov AE, Vasilenko EK, et al. Lung cancer risks from plutonium: an updated analysis of data from the Mayak worker cohort. Radiat Res. 2013;179:332-342.

13. Kudo S, Ishida J, Yoshimoto K, Furuta H, Kasagi F. Differences in the methods used in radiation epidemiological cohort studies among nuclear workers in each country. Hoken Butsuri (Online). 2017;52:265-274.

14. Jacob P, Ruhm W, Walsh L, Blettner M, Hammer G, Zeeb H. Is cancer risk of radiation workers larger than expected? Occup Environ Med. 2009;66:789-796.

15. Shore R, Walsh L, Azizova T, Ruhm W. Risk of solid cancer in low dose-rate radiation epidemiological studies and the dose-rate effectiveness factor. Int J Radiat Biol. 2017;93:1064-1078.

16. Haley BM, Paunesku T, Grdina DJ, Woloschak GE. The increase in animal mortality risk following exposure to sparsely ionizing radiation is not linear quadratic with dose. PLoS One. 2015;10: e0140989.

17. Tran V, Little MP. Dose and dose rate extrapolation factors for malignant and non-malignant health endpoints after exposure to gamma and neutron radiation. Radiat Environ Biophys. 2017; 56:299-328.

18. Niwa O, Barcellos-Hoff MH, Globus RK, Harrison JD, Hendry JH, Jacob P, et al. ICRP Publication 131: stem cell biology with respect to carcinogenesis aspects of radiological protection. Ann ICRP. 2015;44:7-357.

19. Conversion coefficients for use in radiological protection against external radiation: adopted by the ICRP and ICRU in September 1995. Ann ICRP. 1996;26:1-205.

20. Menzel HG, Clement C, DeLuca P. ICRP Publication 110. Realistic reference phantoms: an ICRP/ICRU joint effort. A report of adult reference computational phantoms. Ann ICRP. 2009;39: $1-164$.

21. Petoussi-Henss N, Bolch WE, Eckerman KF, Endo A, Hertel N, Hunt J, et al. ICRP Publication 116: conversion coefficients for radiological protection quantities for external radiation exposures. Ann ICRP. 2010;40:1-257.

22. Paquet F, Etherington G, Bailey MR, Leggett RW, Lipsztein J, Bolch WE, et al. ICRP Publication 130: occupational Intakes of Radionuclides: Part 1. Ann ICRP. 2015;44:5-188.

23. Paquet F, Bailey MR, Leggett RW, Lipsztein J, Fell TP, Smith T, et al. ICRP Publication 134: occupational Intakes of Radionuclides: Part 2. Ann ICRP. 2016;45:7-349.

24. Paquet F, Bailey MR, Leggett RW, Lipsztein J, Marsh J, Fell TP, et al. ICRP Publication 137: occupational Intakes of Radionuclides:
Part 3. Ann ICRP. 2017;46:1-486.

25. Bolch WE, Jokisch D, Zankl M, Eckerman KF, Fell T, Manger R, et al. ICRP Publication 133: the ICRP computational framework for internal dose assessment for reference adults: specific absorbed fractions. Ann ICRP. 2016;45:5-73.

26. Thierry-Chef I, Marshall M, Fix JJ, Bermann F, Gilbert ES, Hacker $\mathrm{C}$, et al. The 15-country collaborative study of cancer risk among radiation workers in the nuclear industry: study of errors in dosimetry. Radiat Res. 2007;167:380-395.

27. Thierry-Chef I, Richardson DB, Daniels RD, Gillies M, Hamra GB, Haylock R, et al. Dose estimation for a study of nuclear workers in France, the United Kingdom and the United States of America: Methods for the International Nuclear Workers Study (INWORKS). Radiat Res. 2015;183:632-642.

28. Task Group on Radiation Quality Effects in Radiological Protection, Committee 1 on Radiation Effects, International Commission on Radiological Protection. Relative biological effectiveness (RBE), quality factor $(\mathrm{Q})$, and radiation weighting factor $\left(\mathrm{W}_{\mathrm{R}}\right)$ : a report of the International Commission on Radiological Protection. Ann ICRP. 2003;33:1-117.

29. Cullings HM, Grant EJ, Egbert SD, Watanabe T, Oda T, Nakamura F, et al. DS02R1: improvements to atomic bomb survivors' input data and implementation of dosimetry system 2002 (DS02) and resulting changes in estimated doses. Health Phys. 2017; 112:56-97.

30. International Commission on Radiological Protection. Human alimentary tract model for radiological protection (ICRP Publication 100: a report of the International Commission on Radiological Protection). Ann ICRP. 2006;36:25-327.

31 . Human respiratory tract model for radiological protection: a report of a Task Group of the International Commission on Radiological Protection. Ann ICRP. 1994;24:1-482.

32. Eckerman K, Endo A. ICRP Publication 107: nuclear decay data for dosimetric calculations. Ann ICRP. 2008;38:7-96.

33. Individual monitoring for intakes of radionuclides by workers: design and interpretation: a report of a Task Group of Committee 4 of the International Commission on Radiological Protection. Ann ICRP. 1988;19:1-315.

34. 1990 Recommendations of the International Commission on Radiological Protection. Ann ICRP. 1991;21:1-201.

35. Satoh D, Takahashi F, Shigemori Y, Sakamoto K. DOSE-Analyzer: a computer program with graphical user interface to analyze absorbed dose inside a body of mouse and human upon external neutron exposure (No. JAEA-DATA/CODE-2010-004). Tokai-mura, Japan: Japan Atomic Energy Agency; 2010. 